\title{
Funcionalidade global de idosos hospitalizados
}

\author{
Global functionality of hospitalized elderly
}

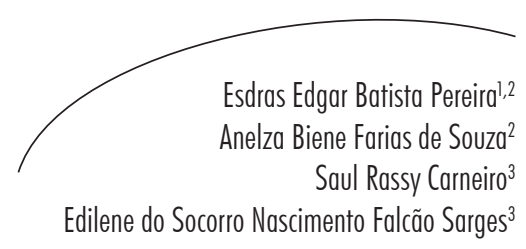

Resumo

Objetivo: Identificar a funcionalidade global de idosos submetidos a internação, correlacionando o desempenho para as atividades de vida diária básicas (ABVD) e instrumentais (AIVD) com os principais sistemas funcionais (cognição, humor, mobilidade e comunicação). Métodos: Trata-se de estudo observacional transversal e analítico com a participação de 94 idosos internados em uma clínica médica. Os instrumentos utilizados foram: Escala de Katz, Escala de Lawton \& Brody, Miniexame do Estado Mental (MEEM), Escala de Depressão Geriátrica 15 (GDS-15), Teste Timed Up and Go (TUG) e a Avaliação Funcional das Habilidades de Comunicação da Associação Americana de Fonoaudiologia (ASHA FACS). Resultados: Foram avaliados 94 idosos, cuja maioria apresentava algum grau de dependência para ABVD (61,71\%) e para AIVD (52,13\%). Nos sistemas funcionais, a autonomia mostrou-se preservada, com desempenho médio de 18,14 pontos no MEEM, e de 4,43 pontos na GDS-15; e a independência, alterada, por desempenho médio de 21,82 segundos no TUG, classificando-os com uma mobilidade regular, e de 5,27 pontos na ASHA FACS, considerando-os com a necessidade de auxílio moderado nessa função. Evidenciou-se moderada e significativa associação entre $\mathrm{O}$ desempenho para as ABVDs e AIVDs com quase todos os sistemas funcionais. Conclusão: A população estudada apresentou funcionalidade global alterada, devido a algum grau de dependência para as ABVDs e AIVDs, com autonomia preservada e independência prejudicada. As correlações evidenciaram que com o decréscimo dos principais sistemas funcionais, ocorreu declínio da funcionalidade global.

\footnotetext{
Programa de Pós-graduação em Oncologia e Ciências Médicas, Núcleo de Pesquisa em Oncologia. Universidade Federal do Pará. Belém, PA, Brasil.

2 Programa de Residência Multiprofissional em Saúde do Idoso, Hospital Universitário João de Barros Barreto. Universidade Federal do Pará. Belém, PA, Brasil.

3 Serviço de Fisioterapia, Hospital Universitário João de Barros Barreto. Universidade Federal do Pará. Belém, PA, Brasil.

Correspondência / Correspondence

Esdras Edgar Batista Pereira

Universidade Federal do Pará - UFPA, Hospital Universitário João de Barros Barreto - HUJBB, Programa

de Residência Multiprofissional em Saúde do Idoso, Serviço de Fisioterapia

Rua dos Mundurucus, 4487, Bairro Guamá, CEP: 66073-000, Belém, PA, Brasil.

E-mail: edgarpereira@ufpa.br
}

Palavras-chave: Idoso.

Hospitalização. Atividades

Cotidianas.Funcionalidade. 


\section{Abstract}

Objective: Identify the global functionality of hospitalized elderly, correlating the performance to basic (BADL) and instrumental (IADL) activities of daily life, with the main functional systems (cognition, mood, mobility and communication). Methods: Analytical, observational, cross-sectional study, with the participation of 94 elderly patients admitted to a medical clinic. The tools used in the assessments were: the Katz Scale, Lawton and Brody Scale, Mini-Mental State Examination (MMSE), Geriatric Depression Scale 15 (GDS-15), Timed Up and Go Test (TUG) and the Functional Assessment of Communication Skills from the American Speech-Language-Hearing Association (ASHA FACS). Results: 94 elderly people were evaluated, where most had some degree of dependence for BADL (61.71\%) and IADL (52.13\%). In functional systems, autonomy proved to be preserved with an average performance of 18.14 points on the MMSE, and 4.43 points in the GDS-15; independence was altered, for average performance of 21.82 seconds on the TUG, classifying them with regular mobility, and 5.27 points in ASHA FACS, considering them as in need of moderate assistance in this task. It was evidenced moderate and significant association between performance for IADL and BADL in nearly all functional systems. Conclusion: The study population presented changed overall functionality, due to some degree of dependence in BADL and IADL, with preserved autonomy and impaired independence. The correlations showed that with the decline of the major functional systems occurred decline in overall functionality.
Key words: Elderly.

Hospitalization. Activities of Daily Living. Functionality.

\section{INTRODUÇÃO}

A saúde do idoso pode ser identificada pela funcionalidade global, definida como a capacidade de gerir a própria vida ou cuidar de si mesmo, influenciada pelo grau de autonomia e independência do indivíduo. ${ }^{1}$ Tais critérios englobam $\mathrm{o}$ funcionamento integrado $\mathrm{e}$ harmonioso das atividades de vida diária com a cognição, humor, mobilidade e comunicação, que permitem dizer se o idoso é saudável ou não, mesmo com alguma doença. ${ }^{2}$

As atividades de vida diárias são tarefas que permitem avaliar a prevalência e a tendência de deficiência. ${ }^{3}$ Para a classificação da funcionalidade do idoso, essas tarefas podem ser identificadas por meio de duas categorias: as atividades básicas de vida diária (ABVDs) e as instrumentais de vida diária (AIVDs). ${ }^{4}$

As ABVDs referem-se às tarefas do cotidiano necessárias para o cuidado com o corpo, como tomar banho, vestir-se, higiene pessoal, transferência, continência esfincteriana e alimentar-se. ${ }^{2}$ As AIVDs são mais complexas e se referem ao cuidado doméstico, como preparo de alimentos, fazer compras, controle do dinheiro, uso do telefone, trabalhos domésticos, lavar e passar roupa, uso correto dos medicamentos e sair de casa. ${ }^{2}$

A hospitalização é reconhecida como um fator de risco para o declínio funcional das pessoas idosas, devido à perda de dependência e autonomia, podendo potencializar um comprometimento funcional já existente, devido à má nutrição, repouso excessivo, privação de sono e a polifarmácia. ${ }^{5}$ Estima-se que esse declínio atinja de $25 \%$ a $35 \%$ dos idosos submetidos a internação hospitalar. ${ }^{6}$

A identificação das variáveis da funcionalidade global do idoso é um processo interdisciplinar, considerada como ferramenta que auxilia na eficácia diagnóstica, na detecção dos problemas de saúde que mais afetam a qualidade de vida do idoso, como as síndromes geriátricas, e na elaboração de planos de cuidados que definem a atuação específica da equipe multiprofissional, tanto no âmbito ambulatorial quanto hospitalar. ${ }^{7,8}$

O objetivo deste estudo foi identificar a funcionalidade global de idosos submetidos a hospitalização, correlacionando o desempenho para as atividades de vida diárias (ABVDs e AIVDs) com os principais sistemas funcionais (cognição, humor, mobilidade e comunicação). 


\section{MÉTODOS}

Foi realizado estudo observacional transversal analítico, com idosos internados na Clínica Médica do Hospital Universitário João de Barros Barreto (HUJBB), em Belém do Pará, no período de março a julho de 2012, aprovado pelo Comitê de Ética em Pesquisa do HUJBB, protocolo $\mathrm{n}^{\mathrm{o}}$ 1.007/11, de acordo com a Resolução do Conselho Nacional de Saúde no 196/96.

\section{Amostra}

No período da pesquisa, foram internados112 idosos (44 homens e 68 mulheres). Estes foram convidados a participar do estudo e realizar a avaliação, visando identificar as condições dos critérios de inclusão e exclusão.

Os critérios de inclusão foram: idade igual ou superior a 60 anos, com condições clínicas favoráveis (normotenso, normocárdico, eupineico e normotérmico) para submissão aos instrumentos avaliativos e que demonstraram interesse em participar do estudo, por meio da assinatura do Termo de Consentimento Livre e Esclarecido. Destes, 18 idosos contemplaram os critérios de exclusão: necessidade de assistência com cuidados intensivos anterior ao momento avaliativo (10 idosos) e ausência do acompanhante no momento da avaliação (oito idosos). A amostra final foi composta por 94 idosos (37 homens e 57 mulheres).

\section{Instrumentos avaliativos}

Os idosos foram submetidos à avaliação multidimensional, que incluiu a análise dos dados demográficos (sexo, idade e escolaridade), clínico-epidemiológicos (impressão diagnóstica e momento da avaliação), do desempenho para capacidade funcional global e dos principais sistemas funcionais.

Avaliação da capacidade funcional global: a capacidade funcional global foi avaliada por meio das atividades de vida diárias utilizando a
Escala de Katz e a Escala de Lawton \& Brody. A Escala de Katz (atividades básicas de vida diária - ABVDs), que se refere às tarefas necessárias para o cuidado com corpo ou autopreservação, composta por seis domínios, com um escore total que varia de 6 a 18 pontos, permite classificar o idoso como independente (6 pontos), semidependente (7 a 16 pontos) e dependente (acima de 16 pontos). ${ }^{2,9}$ A Escala de Lawton \& Brody (atividades instrumentais de vida diária - AIVDs), que se refere às tarefas necessárias para o cuidado com o domicílio ou atividades domésticas, composta por nove domínios, com um escore total que varia de 9 a 27 pontos, permite classificar o idoso como dependente ( 9 pontos), semidependente (10 a 18 pontos) e independente (19 a 27 pontos). ${ }^{2,10}$

Avaliação cognitiva: a função cognitiva foi avaliada por meio do Miniexame do Estado Mental (MEEM), composto por 11 domínios (orientação temporal, orientação espacial, registro, atenção e cálculo, memória de evocação, nomear objetos, repetição, comandos, escrita, ler e executar e copiar), com o escore total que varia de 0 a 30 pontos. É um teste de rastreio que possibilita classificar o idoso como normal ou com possível demência, considerando sua escolaridade, com escore de normalidade de 14 pontos para analfabetos, 18 pontos para aqueles entre 1 e 8 anos de estudo e 24 pontos para aqueles com mais de 8 anos de estudo. ${ }^{11}$

Avaliação do humor: o humor foi avaliado pela Escala de Depressão Geriátrica de Yesavage 15 (GDS-15), composta por 15 perguntas, com escore total que varia de 0 a 15 pontos. Através dessa escala, pode-se obter o resultado dentro da normalidade ( 0 a 5 pontos), com depressão (6 a 10 pontos) ou com depressão grave (11 a 15 pontos). ${ }^{12}$

Avaliação da mobilidade: a mobilidade foi analisada por meio do Teste Time Up and Go (TUG), onde o idoso, inicialmente sentado em uma cadeira com assento a 45 centímetros do chão, foi submetido a um trajeto de três metros de ida e volta, sendo devidamente cronometrado e classificado conforme o 
tempo de desempenho: mobilidade normal (menor que 10 segundos); boa mobilidade (11 a 20 segundos); mobilidade regular (21 a 30 segundos) e mobilidade prejudicada (acima de 30 segundos)..$^{13}$

Avaliação da funcionalidade da comunicaşão: a funcionalidade da comunicação foi investigada utilizando a análise quantitativa da Avaliação Funcional das Habilidades de Comunicação da Associação Americana de Fonoaudiologia (ASHA FACS), composta por 26 questões, adequadas a todos os idosos entrevistados, divididas em quatro domínios, com um escore total que varia de 1 a 7 pontos, permitindo classificar o idoso como sem auxílio (7 pontos), auxílio moderado (3 a 6 pontos) e auxílio máximo (1 a 2 pontos). ${ }^{14}$

\section{Análise estatística}

Para análise dos dados, foi realizada a análise descritiva dos dados, utilizando a frequência absoluta, média, desvio-padrão e mediana. Para verificar a normalidade da distribuição dos dados, foi utilizado o teste D'Agostino-Pearson.

A associação entre as variáveis da capacidade funcional (ABVD e AIVD) e as dos sistemas funcionais (MEEM, GDS-15, TUG e ASHA FACS) foi avaliada por método não paramétrico, correlação de Spearman, visto que todas as variáveis não apresentaram distribuição gaussiana. A correlação foi avaliada mediante seu coeficiente (rs), como forte $(0,70$ a 1,0), moderada $(0,3$ a 0,7$)$ e fraca $(0$ a 0,3$)$. No processamento estatístico, foi utilizado o software Bioestat 5.4. Foi previamente fixado o nível de significância de 5\% $(\mathrm{p}<0,05)$.

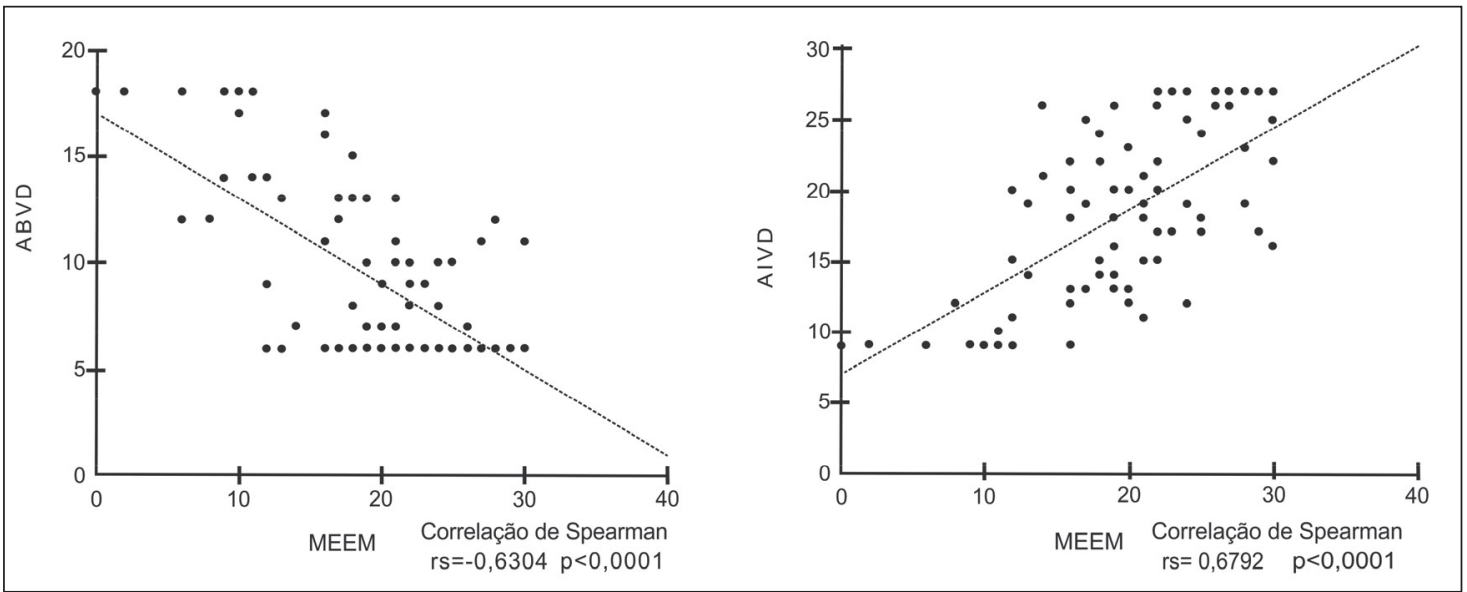

Figura 1. Correlação entre o Miniexame do Estado Mental (MEEM) e o desempenho para Atividades Básicas e Instrumentais de Vida Diária (ABVD e AIVD) dos idosos internados na clínica médica do HUJBB. Belém, PA, 2012. 

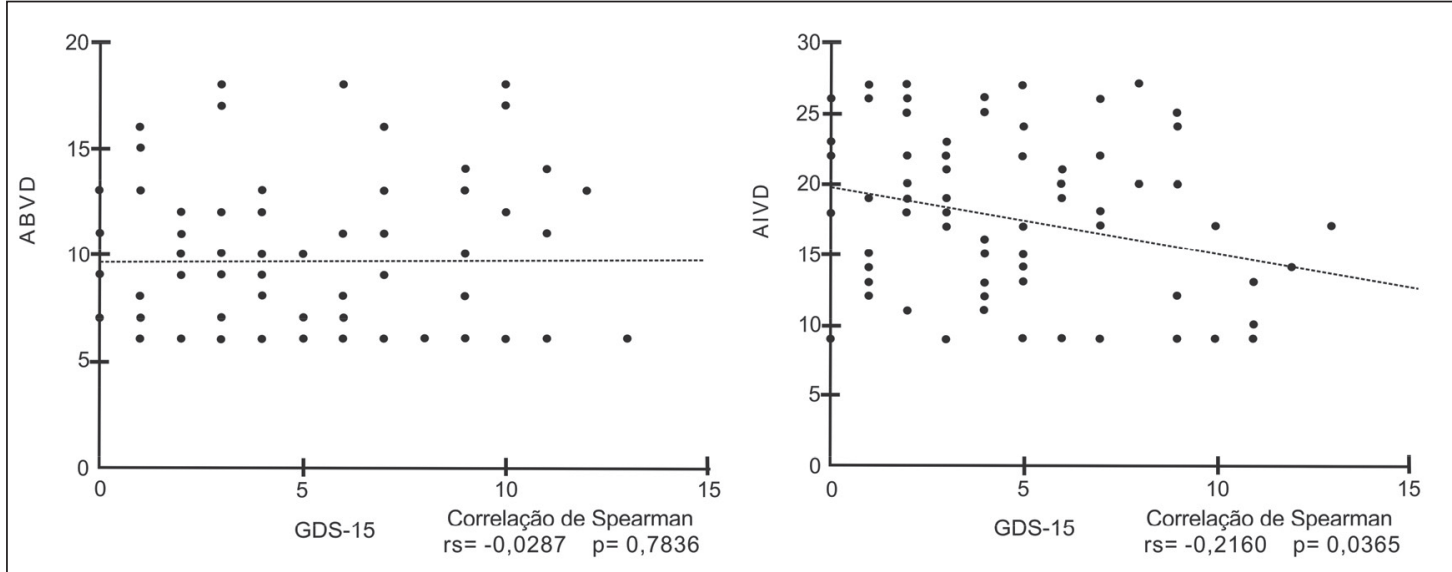

Figura 2. Correlação entre a Escala de Depressão Geriátrica de Yesavage 15 (GDS-15) e o desempenho para Atividades Básicas e Instrumentais de Vida Diária (ABVD e AIVD) dos idosos internados na clínica médica do HUJBB. Belém, PA, 2012.
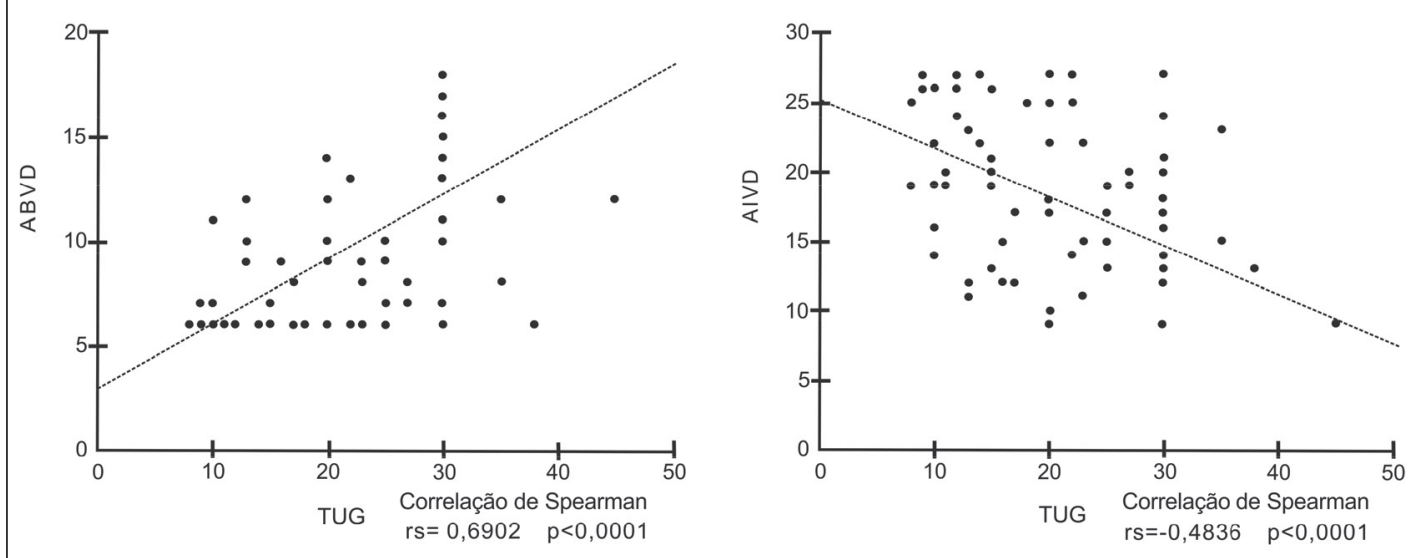

Figura 3. Correlação entre o Teste Time Up and Go (TUG) e o desempenho para Atividades Básicas e Instrumentais de Vida Diária (ABVD e AIVD) dos idosos internados na clínica médica do HUJBB. Belém, PA, 2012.
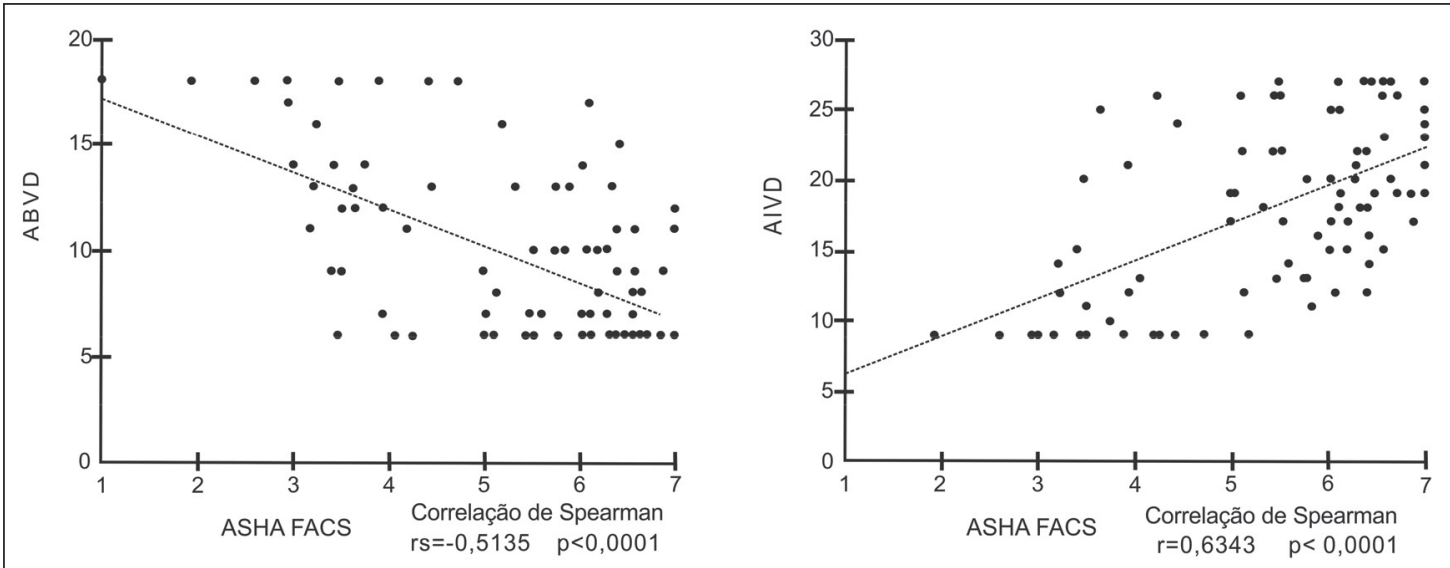

Figura 4. Correlação entre a Avaliação Funcional das Habilidades de Comunicação da Associação Americana de Fonoaudiologia (ASHA FACS) e o desempenho para Atividades Básicas e Instrumentais de Vida Diária (ABVD e AIVD) dos idosos internados na clínica médica do HUJBB. Belém, PA, 2012. 


\section{RESULTADOS}

Participaram desta pesquisa 94 idosos, com uma média de idade de 71,32 ( $\pm 8,70)$ anos, predominantemente do sexo feminino $(60,64 \%)$, com tempo médio de estudo de 4,10 $( \pm 3,10)$ anos. Além disso, observou-se que os motivos das internações foram por doença cardiovascular $(67,02 \%)$, doença do sistema digestivo $(54,26 \%)$ e doença endócrina e metabólica (50,00\%), dentre outras e suas associações, com um tempo médio de internação de quatro dias no momento avaliativo (tabela 1 ).

A investigação do perfil da capacidade funcional mostrou que a maioria era semidependente ou dependente para as atividades de vida diária, sejam básicas ou instrumentais (tabela 2).

Tabela 1. Perfil dos idosos internados na clínica médica do HUJBB. Belém-PA, 2012.

\begin{tabular}{|c|c|c|}
\hline Variáveis & Frequência & $\%$ \\
\hline \multicolumn{3}{|l|}{ Sexo } \\
\hline Masculino & 37 & 39,36 \\
\hline Feminino & 57 & 60,64 \\
\hline \multicolumn{3}{|l|}{ Idade (anos) } \\
\hline $60-74$ & 64 & 68,09 \\
\hline $75-89$ & 28 & 29,79 \\
\hline $90-104$ & 2 & 2,13 \\
\hline \multicolumn{3}{|l|}{ Escolaridade } \\
\hline Analfabeto & 19 & 20,21 \\
\hline Entre 1 e 8 anos de estudo & 68 & 72,34 \\
\hline Mais de 8 anos de estudo & 7 & 7,45 \\
\hline \multicolumn{3}{|l|}{ Impressão diagnóstica } \\
\hline Doença cardiovascular & 63 & 67,02 \\
\hline Doença do sistema digestivo & 51 & 54,26 \\
\hline Doença endócrina e metabólica & 47 & 50,00 \\
\hline Doença osteomuscular & 30 & 31,91 \\
\hline \multicolumn{3}{|l|}{ Momento da avaliação } \\
\hline $1^{a}$ semana de internação & 87 & 92,55 \\
\hline $2^{\mathrm{a}}$ semana de internação & 2 & 2,13 \\
\hline $3^{a}$ semana de internação & 2 & 2,13 \\
\hline $4^{a}$ semana de internação & 3 & 3,19 \\
\hline Total & 94 & 100,00 \\
\hline
\end{tabular}


Tabela 2. Perfil da capacidade funcional dos idosos internados. Belém-PA, 2012.

\begin{tabular}{lcc}
\hline \multicolumn{1}{c}{ Variáveis } & Frequência & $\%$ \\
\hline ABVD (Escala de Kat₹) & 36 & 38,30 \\
Independente & 46 & 48,94 \\
Semidependente & 12 & 12.77 \\
Dependente & & \\
AIVD (Escala de Lawton \& Brody) & 45 & 47,87 \\
Independente & 31 & 32,98 \\
Semidependente & 18 & 19,15 \\
Dependente & 94 & 100,00 \\
\hline Total &
\end{tabular}

$\mathrm{ABVD}=$ atividade básica de vida diária; $\mathrm{AIVD}=$ atividade instrumental de vida diária.

No desempenho médio para as atividades de vida diária, evidenciaram-se $9,74( \pm 4,20)$ pontos para as ABVDs e 17,69 $( \pm 6,34)$ para as AIVDs. Nos testes dos sistemas funcionais, observou-se um desempenho médio de 18,14 $( \pm 7,55)$ pontos no teste de cognição (MEEM); 4,43 ( $\pm 3,37$ ) pontos no teste do humor (GDS-15); 21,82
$( \pm 8,65)$ segundos no teste da mobilidade (TUG); e $5,27( \pm 2,23)$ pontos no teste da comunicação (ASHA FACS) (tabela 3).

Os dados referentes às variáveis da capacidade funcional global do idoso foram correlacionados com cada sistema funcional, permitindo a análise da associação entre os mesmos (tabela 4).

Tabela 3. Características da capacidade funcional e dos sistemas funcionais dos idosos internados. Belém-PA, 2012.

\begin{tabular}{lccc}
\hline \multicolumn{1}{c}{ Variáveis } & Média $( \pm \mathrm{dp})$ & Mediana & D’Agostino-Pearson p-valor \\
\hline Capacidade funcional & & & \\
ABVD (Escala de Katz) & $9,74( \pm 4,20)$ & 8,00 & $<0,05$ \\
AIVD (Escala de Lawton \& Brody) & $17,69( \pm 6,34)$ & 18,00 & $<0,05$ \\
& & & \\
Sistemas funcionais & $18,14( \pm 7,55)$ & 19,50 & $<0,05$ \\
Cognição (MEEM) & $4,43( \pm 3,37)$ & 4,00 & $<0,05$ \\
Humor (GDS-15) & $21,82( \pm 8,65)$ & 22,00 & $<0,05$ \\
Mobilidade (TUG) & $5,27( \pm 1,50)$ & 5,76 & $<0,05$ \\
Comunicação (ASCHA FACS) &
\end{tabular}

$\mathrm{ABVD}=$ atividade básica de vida diária; $\mathrm{AIVD}=$ atividade instrumental de vida diária; $\mathrm{dp}=$ desvio-padrão; MEEM= Miniexame do Estado Mental; GDS-15= Escala de Depressão Geriátrica de Yesavage 15; TUG= Teste Timed Up And Go; ASHA FACS= Avaliação Funcional das Habilidades de Comunicação da Associação Americana de Fonoaudiologia. 
Tabela 4. Correlação entre o desempenho na capacidade funcional (ABVD e AIVD) e os sistemas funcionais dos idosos internados. Belém-PA, 2012.

\begin{tabular}{lccc}
\hline \multicolumn{1}{c}{ Atividades de Vida Diária } & Sistemas Funcionais & rs & p-valor \\
\hline \multirow{2}{*}{ ABVD (Escala de Katz) } & Cognição (MEEM) & $-0,6304$ & $<0,0001$ \\
& Humor (GDS-15) & $-0,0287$ & 0,7836 \\
& Mobilidade (TUG) & 0,6902 & $<0,0001$ \\
& Comunicação (ASHA FACS) & $-0,5135$ & $<0,0001$ \\
AIVD (Escala de Lawton \& & Cognição (MEEM) & 0,6792 & $<0,0001$ \\
Brody) & Humor (GDS-15) & $-0,2160$ & 0,0365 \\
& Mobilidade (TUG) & $-0,4836$ & $<0,0001$ \\
& Comunicação (ASHA FACS) & 0,6343 & $<0,0001$ \\
\hline
\end{tabular}

$\mathrm{ABVD}=$ atividade básica de vida diária; $\mathrm{AIVD}=$ atividade instrumental de vida diária; MEEM= Miniexame do Estado Mental; GDS-15= Escala de Depressão Geriátrica de Yesavage 15; TUG= Teste Timed Up And go; ASHA FACS= Avaliação Funcional das Habilidades de Comunicação da Associação Americana de Fonoaudiologia; rs= coeficiente da correlação de Spearman.

Nos sistemas funcionais referentes à autonomia (cognição e humor), a correlação entre as atividades de vida diária e a cognição demonstrou uma associação moderada e significativa. Quanto menor a pontuação no MEEM, maior a pontuação para as ABVDs e menor para as AIVDs. A correlação entre as atividades de vida diária e o humor demonstrou uma associação fraca, significante somente para as AIVDs. Quanto menor a pontuação no GDS15, maior a pontuação para as AIVDs (tabela 4).

Nos sistemas funcionais referentes à independência (mobilidade e comunicação), a correlação entre as atividades de vida diária e a mobilidade demonstrou uma associação moderada e significante. Quanto menor a pontuação no TUG, menor a pontuação para as ABVDs e maior para as AIVDs. A correlação entre as atividades de vida diária e a comunicação demonstrou associação moderada e significante. Quanto menor a pontuação no ASHA FACS, maior a pontuação para as ABVDs e menor para as AIVDs (tabela 4).

\section{DISCUSSÃO}

Os participantes da pesquisa apresentaram características que corroboram Motta et al., ${ }^{15}$ que ao estudarem 767 idosos de um hospital público de Petrópolis-RJ, identificaram uma população predominantemente feminina $(60,50 \%)$ com idade entre 60 e 79 anos (77,83\%). Esmayel et al. ${ }^{16}$ estudaram um grupo de 200 idosos hospitalizados e observaram resultados similares, que diferiram somente na frequência dos gêneros, com maior ocorrência do gênero masculino (56\%).

Notou-se que a maioria dos idosos foi submetida à avaliação da capacidade funcional global ainda na primeira semana de internação $(92,55 \%)$, o que sugere um estado funcional prévio à hospitalização, já exposto a diversos fatores de risco. Estima-se que parte significativa do declínio funcional ocorra poucos dias antes da internação. ${ }^{17}$

Os dados da capacidade funcional global permitiram observar que a maioria dos internados 
apresentou algum grau de dependência para as ABVDs $(61,71 \%)$ e para as AIVDs $(52,13 \%)$. Esse predomínio de idosos dependentes também foi identificado por Chang et al.: ${ }^{18} 68,8 \%$ dos idosos foram dependentes para as ABVDs e em $78,7 \%$ para as AIVDs. Levando em consideração que em ambos os estudos a maior parte dos idosos foi avaliada ainda na primeira semana de internação, acredita-se em um declínio funcional anterior à hospitalização. ${ }^{19}$

Segundo Lee et al., ${ }^{20}$ o nível funcional já comprometido nos primeiros dias de internação pode associar-se com idade avançada, gravidade das comorbidades instaladas e estado cognitivo, podendo ser potencializado com as práticas hospitalares (assistência não especializada, imobilidade no leito, uso de psicofármacos, aplicação de sonda vesical, etc.).

Como os fatores associados ao comprometimento funcional variam entre as populações, outros estudos encontraram resultados distintos, como os de Martin \& Jentoft ${ }^{21}$ e Banouby et al..$^{22}$, que estudaram grupos distintos de idosos hospitalizados e observaram frequência de dependência maior para as AIVDs do que para as ABVDs.

A identificação do perfil funcional faz parte do processo de diagnóstico, tanto na definição dos déficits relacionados à saúde quanto na avaliação dos declínios da funcionalidade do idoso, sendo ambos necessários para embasar estudos epidemiológicos e populacionais, e também para compreender o idoso no nível individual, levando em consideração aspectos clínicos, biológicos e funcionais. ${ }^{23}$

O estado funcional do idoso pode ser bem compreendido a partir da análise dos principais sistemas funcionais, por meio da autonomia e da independência. Para Carretta et al., ${ }^{24}$ a complexidade do ambiente hospitalar produz uma realidade diferente ao idoso, que, fragilizado pela doença, sente-se mais impotente.
A associação desses sistemas com funcionalidade deve fazer parte da identificação de um possível declínio funcional.

Ao analisar a autonomia dos idosos, observouse um desempenho médio de 18,14 pontos na função cognitiva e de 4,43 pontos para o humor, ambos dentro da normalidade, acreditandose que, em média, os idosos possuíam sua autonomia preservada. Mas ao estratificar essas variáveis, pode-se observar que 32,98\% dos idosos apresentaram possível comprometimento cognitivo e risco para depressão, realidade similar à encontrada em quatro hospitais da Austrália e em 12 hospitais de Taiwan. ${ }^{18,25}$

A associação do MEEM com as atividades diárias foi moderada, evidenciando que com o declínio da função cognitiva houve declínio do desempenho para ABVDs e AIVDs. Já a correlação entre o humor $\mathrm{e}$ as atividades de vida diária evidenciou mais significativa associação com as AIVDs, resultado justificado por Santos et al., ${ }^{26}$ que afirmaram que idosos depressivos apresentam maior tendência ao comprometimento físico, social e funcional.

Esses dados demonstram que a utilização dos instrumentos de rastreio da função cognitiva e do humor, MEEM e GDS-15, são importantes na prática profissional em saúde do idoso, devendo fazer parte da rotina hospitalar, como preditores da redução da funcionalidade global do idoso hospitalizado, para o amparo preventivo e de programas de reabilitação de forma mais precoce.

No que tange à independência, observouse um desempenho médio de 21,82 segundos no teste da mobilidade, classificando-os com uma mobilidade regular, e de 5,27 pontos para comunicação, considerando-os com a necessidade de auxílio moderado nessa função. As habilidades da comunicação são fundamentais no ambiente hospitalar, pois sua manutenção facilita a ideal interação entre o paciente e a equipe de saúde. ${ }^{27}$ 
Mais especificamente, $52,13 \%$ dos idosos possuíam a mobilidade regular ou prejudicada. A mobilidade alterada também foi encontrada no estudo de Chen et al., ${ }^{28}$ frequente em 83,8\% dos 117 idosos de um hospital geral de Taiwan. Escores de desempenho no TUG similares ao presente estudo foram observados por Trombetti et al. $^{29}$ e Said et al., ${ }^{30}$ variando entre 26 e 31 segundos em média, dados que evidenciam um prejuízo funcional na mobilidade nos primeiros dias de hospitalização.

Foi observada moderada associação entre a mobilidade e as atividades de vida diária, o que permite afirmar que, com a mobilidade prejudicada, houve declínio das ABVDs e das AIVDs, assim como na habilidade comunicativa, que expõe a significativa associação com ABVDs e AIVDs.

Os idosos avaliados apresentaram certo grau de dependência funcional, e devido a isso são expostos a possíveis complicações durante o período de internação, como imobilidade no leito, transtornos cognitivos, riscos de queda, entre outras. Com a identificação do nível funcional e dos parâmetros clínicos dos idosos hospitalizados, pode-se ter conhecimento de fatores de risco para o tempo de internação, reinternação, dependência após alta hospitalar, institucionalização e para a mortalidade nessa população, na qual os idosos mais dependentes são os mais vulneráveis a esses fatores. ${ }^{31}$

A autonomia e a independência são as variáveis determinantes para o bom desempenho das atividades de vida diária, tendo associação direta com os sistemas funcionais. As alterações da autonomia observadas em um terço dos idosos estudados podem ter origem na condição prévia à internação, ligadas a baixa escolaridade, idade avançada, comorbidades, administração de drogas psicofármacos, má nutrição, estilo de vida, insuficiência familiar e suporte social reduzido. ${ }^{32}$

O rastreio da função cognitiva e do humor demonstra importância para o planejamento dos cuidados do idoso após a hospitalização. Helvik et al. $^{33}$ identificaram que, após a internação hospitalar, ocorre um declínio funcional, independentemente do estado funcional prévio, mas associados à redução da cognição e do humor, refletindo sobre a saúde global e qualidade de vida do idoso.

A piora da funcionalidade antes da admissão hospitalar pode influenciar o declínio funcional durante a hospitalização, que em geral ocorre devido ao aparecimento de uma doença aguda, agravamento de doenças crônicas ou uma nova condição de comprometimento funcional, que podem ser o motivo da internação hospitalar. ${ }^{34}$

Os doentes que não conseguem manter a homeostase funcional durante e após a hospitalização se tornam mais frágeis. ${ }^{35} \mathrm{O}$ rastreio adequado prevê o declínio funcional resultado da hospitalização, que deve ser identificado logo após a admissão.

O idoso hospitalizado necessita da assistência de uma equipe multiprofissional integrada e conhecedora das principais ferramentas de rastreio para a identificação do declínio funcional, possivelmente presente na admissão ou no decorrer do período de internação.

É necessário mencionar que somente as variáveis estudadas não foram suficientes para o entendimento completo da funcionalidade do idoso, pois se faz necessário investigar outros domínios não abordados no estudo, como o estado nutricional, investigação de polifarmácia, riscos sociofamiliares, entre outros. Outra limitação da pesquisa foi a não homogeneização do momento avaliativo, deixando-os sujeitos a avaliação após possíveis ações iatrogênicas da equipe de saúde.

Abordagens similares a este estudo se mostram importantes no contexto clínico, pois a utilização dos instrumentos avaliativos pode contribuir significativamente na elaboração de planos de cuidados para assistência ao idoso hospitalizado. Através da identificação da funcionalidade global, norteiam-se medidas que reduzam o comprometimento funcional adquirido antes da internação e previnam o oriundo do processo de hospitalização, reduzindo os riscos de complicações e melhorando a qualidade de vida do idoso. 


\section{CONCLUSÃO}

A população estudada apresentou sua funcionalidade global alterada, devido a algum grau de dependência para as atividades de vida diária. Os idosos foram semidepentendes para atividades de vida diária, com autonomia preservada, e independência prejudicada devido à mobilidade regular e a necessidade moderada de auxílio para comunicação.

Foi possível identificar moderada e significativa associação dessas atividades com quase todos os sistemas funcionais abordados.

\section{REFERÊNCIAS}

1. Freitas RS, Fernandes MH, Coqueiro RS, Reis Júnior WM, Rocha SV, Brito TA. Capacidade funcional e fatores associados em idosos: estudo populacional. Acta Paul Enferm 2012;25(6):933-9.

2. Moraes EN. Atenção à saúde do Idoso: Aspectos Conceituais. Brasília ,DF: Organização PanAmericana da Saúde, Representação Brasil; 2012.

3. Chan KS, Kasper JD, Brandt J, Pezzin LE. Measurement Equivalence in ADL and IADL Difficulty Across International Surveys of Aging: findings from the HRS, SHARE, and ELSA.J Gerontol Ser B Psychol Sci Soc Sci 2012;67(1):121-32.

4. Ramos LR, Andreoni S, Coelho Filho JM, Costa MFL, Matos DL, Rebouças M, et al. Perguntas mínimas para rastrear dependência em atividades da vida diária em idosos. Rev Saúde Pública 2013;47(3):506-13.

5. Palleschi L, Alfieri W, Salani B, Fimognari FL, Marsilii A, Pierantozzi A, et al. Functional Recovery of Elderly Patients Hospitalized in Geriatric and General Medicine Units. The Progetto Dimissioni in Geriatria Study. J Am Geriatr Soc 2011;59(2):193-9.

6. Sales MVC, Silva TJA, Gil Júnior LA, Jacob Filho W. Efeitos adversos da internação hospitalar para o idoso. Geriatr Gerontol 2010;4(4):238-46.

7. Netto MP. O Estudo da Velhice: histórico do envelhecimento e da velhice. In: Freitas EV, Py L, Cançado FAX, Doll J, Gorzoni ML. Tratado de Geriatria e Gerontologia. 3 ed. Rio de Janeiro: Guanabara Koogan; 2011. p. 3-13.

8. Moraes EN, Marino MCA, Santos RR. Principais síndromes geriátricas. Rev Med Minas Gerais 2010;20(1):54-66.
Com isso, evidenciou-se que, com o declínio dos principais sistemas funcionais, ocorreu declínio da funcionalidade global, o que reforça os achados da literatura, que mencionam a necessidade da compreensão dos sistemas funcionais na identificação da funcionalidade global e da saúde do idoso.

\section{AGRADECIMENTOS}

Ministério da Educação, Universidade Federal do Pará e Serviço de Fisioterapia do Hospital Universitário João de Barros Barreto (HUJBB).

9. Afonso MS, Silveira KS, Soares MP, Lopes JF, Azevedo P, Brito LCN. Déficits funcionais de idosos correlacionados a cada década de vidas. Rev Inspirar Mov Saúde 2013;5(2):1-6.

10. Palumbo MRB, Daussy MFS; Prefeitura de Florianópolis, Secretaria Municipal de Saúde, Diretoria da Atenção Primária a Saúde. Protocolo de Atenção a Saúde do Idoso. Tubarão: Copiart; 2011.

11. Gontijo JV. Estado cognitivo e funcional de idosos de uma instituição de longa permanência do município de Divinópolis-MG [dissertação]. Divinópolis, MG: Universidade do Estado de Minas Gerais; 2010.

12. Prata HL, Alves Júnior ED, Paula FL, Ferreira SM. Envelhecimento, depressão e quedas: um estudo com os participantes do Projeto Prev-Quedas. Fisioter Mov 2011;24(3):437-43.

13. Bretan O, Silva Júnior JE, Ribeiro OR, Corrente JE. Risk of falling among elderly persons living in the community: assessment by the Timed up and go test. Braz J Otorhinolaryngol 2013;79(1):18-21.

14. Carvalho IAM. Avaliação funcional das habilidades da comunicação: ASHA FACS para população com doença de Alzheimer [tese]. São Paulo: Universidade de São Paulo, Faculdade de Medicina; 2006.

15. MottaI CCR, HanselI CG, SilvaI J. Perfil de internações de pessoas idosas em um hospital público. Rev Eletr Enf 2010;12(3):471-7.

16. Esmayel EMM, Eldarawy MM, Hassan MMM, Hassanin HM, Ashour WMR, Mahmoud W. Nutritional and Functional Assessment of Hospitalized Elderly: Impact of Sociodemographic Variables. J Aging Res [internet]. 2013 [acesso em 02 set 2013]:1-7. Disponível em: http://dx.doi.org/10.1155/2013/101725 
17. Palleschi L, Fimognari FL, Pierantozzi A, Salani B, Marsilii A, Zuccaro SM, et al. Acute functional decline before hospitalization in older patients. Geriatr Gerontol Int [internet]. 2013 [acesso em 02 set 2013]:1-8. Disponível em: http://onlinelibrary.wiley. com/doi/10.1111/ggi.12160/pdf

18. Chang HH, Tsai SL, Chen XY, Liu WJ. Outcomes of hospitalized elderly patients with geriatric syndrome: report of a community hospital reform plan in Taiwan. Arch Gerontol Geriatr 2010;50 Suppl 1:S30-33.

19. Vos AJBM, Asmus-Szepesi KJE, Bakker TJEM, Vreede PL, Wijngaarden JV, Steyerberg WE, et al. Integrated approach to prevent functional decline in hospitalized elderly: the Prevention and Reactivation Care Program (PReCaP). BMC Geriatr 2012;12(7):1-11.

20. Lee S, Staffileno BA, Fogg L. Influence of staff education on the function of hospitalized elders. Nurs Outlook 2013;61(1):2-8

21. Martın SM, Jentoft AJC. Impact of hospital admission on functional and cognitive measures in older subjects. Eur Geriatr Med 2012;3(4):208-12.

22. Banouby SMHE, Hamza SA, Rahman SAA, Mortagy AK. Predictors of Hospital length of Stay among Egyptian Elderly. Life Sci J 2013;10(4):166-70.

23. Malaguarnera M, Marco V, Frazzetto PM, Motta M What is the frailty in elderly? Value and significance of the multidimensional assessments. Arch Gerontol Geriatr 2013;56(1):23-6.

24. Carretta MB, Bettinelli LA, Erdmann AL. Reflexões sobre o cuidado de enfermagem e a autonomia do ser humano na condição de idoso hospitalizado. Rev Bras Enferm 2011;64(5):958-62.

25. Travers C, Byrne GJ, Pachana NA, Klein K, Gray I. Validation of the interrai cognitive performance scale against independent clinical diagnosis and the mini-mental state examination in older hospitalized patients. J Nutr Health Aging 2013;17(5):435-9.

26. Santos JG, Pereira JR, Teixeira CVL, Corazza DI, Vital TM, Costa JLR. Sintomas depressivos e prejuízo funcional de Idosos de um Centro-Dia. Geriátrico. J Bras Psiquiatr 2012;61(2):102-6.
27. O’Halloran R, Worrall L, Hickson L. Stroke patients communicating their healthcare needs in hospital: a study within the ICF framework. Int J Lang Commun Disord 2012;47(20):130-43.

28. Chen YM, Chuang YW, Liao SC, Lin CS, Yang SH, Tang YJ, et al. Predictors of functional recovery (FR) for elderly hospitalized patients in a geriatric evaluation and management unit (GEMU) in Taiwan. Arch Gerontol Geriatr 2010;50 Suppl 1:S1-5.

29. Trombetti A, Hars M, Herrmann F, Rizzoli R, Ferrari S. Effect of a multifactorial fall-and-fracture risk assessment and management program on gait and balance performances and disability in hospitalized older adults: a controlled study. Osteoporos Int 2013;24(3):867-76

30. Said CM, Morris ME, Woodward M, Churilov L, Bernhardt J. Enhancing physical activity in older adults receiving hospital based rehabilitation: a phase II feasibility study. BMC Geriatr 2012;12(26):1-8.

31. Xu H, Covinsky KE, Stallard E, Thomas J, Sands LP. Insufficient Help for Activity of Daily Living Disabilities and Risk of All-Cause Hospitalization. J Am Geriatr Soc 2012;60(5):927-33.

32. Foroni PM, Santos PL. Fatores de risco e proteção associados ao declínio cognitivo no envelhecimento: revisão sistemática de literatura. Rev Bras promoç Saúde 2012;25(3):364-73.

33. Helvik AS, Selbaek G, Engedl K. Functional decline in older adults one year after hospitalization. Arch Gerontol Geriatr 2013;57(3):305-10

34. Isaia G, Bo M, Aimonino N, Isaia GC, Michelis G, Miceli $\mathrm{C}$, et al. Functional decline two weeks before hospitalization in an elderly population. Aging Clin Exp Res 2010;22(4):352-5.

35. Saint-Hubert M, Schoevaerdts D, Cornette P, D'hoore W, Boland B, Swine C. Predicting functional adverse outcomes in hospitalized older patients: a systematic review of screening tools. J Nutr Health Aging 2010;14(5):394-9. 